\title{
Costs to Health Services and the Patient of Treating Tuberculosis: A Systematic Literature Review
}

\author{
Yoko V. Laurence ${ }^{1}$ - Ulla K. Griffiths ${ }^{1}$ • Anna Vassall ${ }^{1}$
}

Published online: 5 May 2015

(C) The Author(s) 2015. This article is published with open access at Springerlink.com

\begin{abstract}
Background Novel tuberculosis (TB) drugs and the need to treat drug-resistant tuberculosis (DR-TB) are likely to bring about substantial transformations in TB treatment in coming years. An evidence base for cost and cost-effectiveness analyses of these developments is needed.

Objective Our objective was to perform a review of papers assessing provider-incurred as well as patient-incurred costs of treating both drug-susceptible (DS) and multidrugresistant (MDR)-TB.

Methods Five databases (EMBASE, Medline, the National Health Service Economic Evaluation Database, the Cost-Effectiveness Analysis Registry, and Latin American and Caribbean Health Services Literature) were searched for cost and economic evaluation full-text papers containing primary DS-TB and MDR-TB treatment cost data published in peer-reviewed journals between January 1990 and February 2015. No language restrictions were set. The search terms were a combination of 'tuberculosis', 'multidrug-resistant tuberculosis', 'cost', and 'treatment'. In the selected papers, study methods and characteristics, quality indicators and costs were extracted into summary tables according to pre-defined criteria. Results were analysed according to country income groups and for provider costs,
\end{abstract}

Electronic supplementary material The online version of this article (doi:10.1007/s40273-015-0279-6) contains supplementary material, which is available to authorized users.

Yoko V. Laurence

yoko.laurence@1shtm.ac.uk

1 Department of Global Health and Development, Faculty of Public Health and Policy, London School of Hygiene and Tropical Medicine, 15-17 Tavistock Place, London WC1H 9SH, UK patient costs and productivity losses. All values were converted to \$US, year 2014 values, so that studies could be compared.

Results We selected 71 treatment cost papers on DSTB only, ten papers on MDR-TB only and nine papers that included both DS-TB and MDR-TB. These papers provided evidence on the costs of treating DS-TB and MDR-TB in 50 and 16 countries, respectively. In $31 \%$ of the papers, only provider costs were included; $26 \%$ included only patient-incurred costs, and the remaining $43 \%$ estimated costs incurred by both. From the provider perspective, mean DS-TB treatment costs per patient were US\$14,659 in high-income countries (HICs), US\$840 in upper middle-income countries (UMICs), US $\$ 273$ in lower middle-income (LMICs), and US\$258 in low-income countries (LICs), showing a strong positive correlation. The respective costs for treating MDRTB were US\$83,365, US\$5284, US\$6313 and US\$1218. Costs incurred by patients when seeking treatment for DS-TB accounted for an additional $3 \%$ of the provider costs in HICs. A greater burden was seen in the other income groups, increasing the costs of DS-TB treatment by $72 \%$ in UMICs, $60 \%$ in LICs and $31 \%$ in LMICs. When provider costs, patient costs and productivity losses were combined, productivity losses accounted for $16 \%$ in HICs, $29 \%$ in UMICs, $40 \%$ in LMICs and $38 \%$ in LICs.

Conclusion Cost data for MDR-TB treatment are limited, and the variation in delivery mechanisms, as well as the rapidly evolving diagnosis and treatment regimens, means that it is essential to increase the number of studies assessing the cost from both provider and patient perspectives. There is substantial evidence available on the costs of DS-TB treatment from all regions of the world. The patient-incurred costs illustrate that the financial 
burden of illness is relatively greater for patients in poorer countries without universal healthcare coverage.

\section{Key Points for Decision Makers}

Drug-susceptible tuberculosis treatment cost data are available from the perspective of both providers and patients from various settings around the world.

Multidrug-resistant tuberculosis treatment costs are not widely available, particularly not for middle- and low-income countries.

Productivity losses were presented in $57 \%$ of the papers, for both drug-susceptible and multidrugresistant tuberculosis. However, methods used varied widely, reflecting the lack of clear guidelines on the best instrument and methods for this estimation.

\section{Introduction}

The post-2015 World Health Organisation (WHO) End TB strategy 2016-2035 has a vision of a 'world free of tuberculosis (TB) (zero deaths, disease or suffering due to TB)' and a goal of 'ending the global TB epidemic' by 2035, defined as an annual incidence of fewer than ten cases per 100,000 of population [1]. These targets are likely to require scaling-up of high-quality drug-sensitive (DS) TB and drug-resistant (DR) TB treatment, but may stretch the resource capacity of national TB programmes far beyond any previous efforts. Country- and contextspecific economic evaluations and budget impact analyses are essential for decision making, but obtaining timely cost data can be expensive and labour intensive. Assembling a repository of quality-assessed DS-TB and multidrug-resistant (MDR)-TB treatment costs can facilitate these processes and identify gaps for future targeted cost data collection.

Earlier reviews have investigated TB treatment costs, but these are either incomplete or no longer up to date. We identified eight previous reviews on TB treatment costs. In 1997, Fryatt [2] reviewed cost-effectiveness papers of TB treatment programmes, in 2004, Russell [3] reviewed the economic burden of households due to TB, and, in 2011, Verdier et al. [4] reviewed economic evaluations of TB control in high-income countries (HICs). Three reviews were published in 2012: two on patient-incurred TB treatment costs in sub-Saharan Africa [5, 6] and one on MDRTB treatment costs [7]. In 2013, Diel et al. [8] published a review determining the costs of TB in the EU, and, in 2014, Tanimura et al. [9] reviewed papers on patient costs in lowand middle-income countries [9]. This present review complements and synthesises the evidence provided in these previous reviews by including papers from all countries, assessing both DS-TB and MDR-TB costs, and evaluating both provider- and patient-incurred costs.

\section{Methods}

\subsection{Search Strategy and Data Extraction}

Peer-reviewed papers were eligible for inclusion if mean treatment cost estimates of DS-TB or MDR-TB in adults were reported and based on primary data that originated from 1990 or later. Five databases were searched: EMBASE, Medline, the National Health Service Economic Evaluation Database, the Cost-Effectiveness Analysis Registry, and the Latin American and Caribbean Health Sciences Literature. An initial search was conducted in April 2013 and updated in February 2015. Therefore, the search period was from January 1990 to February 2015. Search terms were a combination of 'tuberculosis', 'multidrug-resistant tuberculosis', 'cost', and 'treatment'. The full search strategies are included in the Electronic Supplementary Material (ESM) Online Resource 1. No language restrictions were applied in the search. To assess relevance, abstracts or papers in Spanish were translated by the authors, and abstracts obtained in French, Hungarian and Russian were translated using electronic translation software (Google Translate) [10]. Reference lists of identified reviews were checked for papers that may have been missed by the database search; references cited in retrieved papers were also examined.

Data were independently extracted by two authors. Any discrepancies were resolved by re-evaluation of the paper in question. A data extraction sheet was used, the composition of which was informed by the data-extraction guidelines for economic evaluations in the Centre for Reviews and Dissemination's Guidance for Undertaking Reviews in Health Care [11] and the Cochrane Handbook for Systematic Reviews of Interventions [12]. Variables included the characteristics of the study, as well as providerand patient-incurred costs. The outcome measure was mean treatment costs per patient. For each paper, all cost items reported, such as drugs, hospitalisation, diagnostic tests and productivity loss, were extracted separately and, where relevant, divided into patient-incurred and provider costs. Patient-incurred costs were further divided into direct costs and productivity losses. Direct patient costs were defined as expenses paid by patients for receiving treatment, such as user fees for health facilities or monitoring or diagnostic 
tests, drug expenditures, transportation and other costs, which included food, non-TB drugs, traditional medicine and room and board for patients not resident near the TB treatment facility. Moreover, if costs were aggregated, this total was included in the 'other' category. Productivity losses were defined as the value of paid and unpaid production loss due to time seeking treatment, being ill, or because of premature mortality [13].

Given that our aim was to provide a dataset that best informs the estimation of current TB treatment costs, in papers that compared the costs of more than one treatment delivery strategy, for instance directly observed treatment (DOT) versus self-administered treatment (SAT) [14], we selected the intervention we considered to best reflect the current standard practice in the respective country. This was determined from the paper, or, if not stated, by consulting with TB experts familiar with the respective countries.

\subsection{Data Analysis}

Costs were converted to 2014 values in the local currency and then to US\$ using the International Monetary Fund's average consumer price indices and OANDA's average annual exchange rates $[15,16]$. For papers that did not provide the year of cost data, we used the year prior to the publication date.

Results were presented according to 2013 World Bank country income groups. HICs were classified as those with per capita gross national income (GNI) of US\$12,746 or greater, upper middle-income countries (UMICs) greater than or equal to US\$4126 and less than US\$12,746, lower middle-income countries (LMICs) greater than or equal to US\$1046 and less than US\$4126, and low-income countries (LICs) less than US\$1046 [17].

The relationship between provider costs and country GNI per capita was assessed using Pearson's correlation coefficient.

\subsection{Study Quality Assessment}

Quality assessment focused on methods for estimating and reporting costs; methods used for determining health effects as part of cost-effectiveness studies were not evaluated. Quality appraisal was based on two guidelines; the Consolidated Health Economic Evaluation Reporting Standards (CHEERS) statement [18] and The Tool to Estimate Patient's Costs published by the TB Coalition for Technical Assistance (TBCTA) [19]. Four requirements of the CHEERS statement were used: (1) sources used for resource quantities and unit costs clearly described, (2) dates of estimated resource quantities and unit costs reported, (3) methods for adjusting unit costs to the reporting year and performing currency conversion explained, and (4) mean values for main categories of estimated costs reported [18]. For papers that included patient costs, quality was further evaluated using two requirements of the TBCTA tool: (1) clear description of patient interview procedures given and (2) methods used for valuing productivity losses explained and justified [19]. Additional quality indicators abstracted from all papers were the number of patients included in the study sample in order to provide some indications of representativeness $[20,21]$. We also extracted whether any measures of dispersion, such as standard deviation (SD), around the mean cost values were given [22]. The review protocol was not registered in any systematic review database or registry. Two authors independently assessed paper quality, with disagreements resolved through discussion.

\section{Results}

\subsection{Paper Selection}

The search identified 4899 papers, and 289 papers were included for full-text review, after which 199 papers were excluded (Fig. 1). Of the excluded papers, 42 presented costs of TB screening in schools or in high-risk individuals, such as immigrants, healthcare workers, individuals with HIV or the elderly. Reporting was insufficient in 28 papers, including only presenting selected cost items or providing costs of a national TB programme without mean treatment costs per patient; 11 papers were excluded because the same primary data were used in an already included paper. A total of 90 papers were included in the analysis; 71 were on DS-TB treatment costs only, nine were on MDR-TB only, and ten included the costs of both.

\subsection{Study Characteristics}

For DS-TB and MDR-TB, 50 and 16 countries were represented, respectively (Table 1). In this review we distinguished between paper and study to illustrate where a paper included cost values for two or more countries, which would thereby represent two or more studies in one paper. Therefore, a total of 95 studies were represented in this review. The oldest paper was from 1995; six papers were from 2014 and one was from 2015, as of the February 2015 search. Country income groups were relatively evenly represented; $28 \%$ of the papers were from HICs, $32 \%$ from UMICs, $19 \%$ from LMICs, and $21 \%$ from LICs.

In 51 papers, only one type of TB treatment management was evaluated (for example, ambulatory DOT), while the remaining 39 papers compared two or more strategies in either a cost-comparison or a cost-effectiveness analysis.

In $31 \%$ of the papers, only provider costs were included, in $26 \%$ only costs incurred by patients, and in the 
Fig. 1 Literature review flow chart. $M D R$ multidrug-resistant, $T B$ tuberculosis

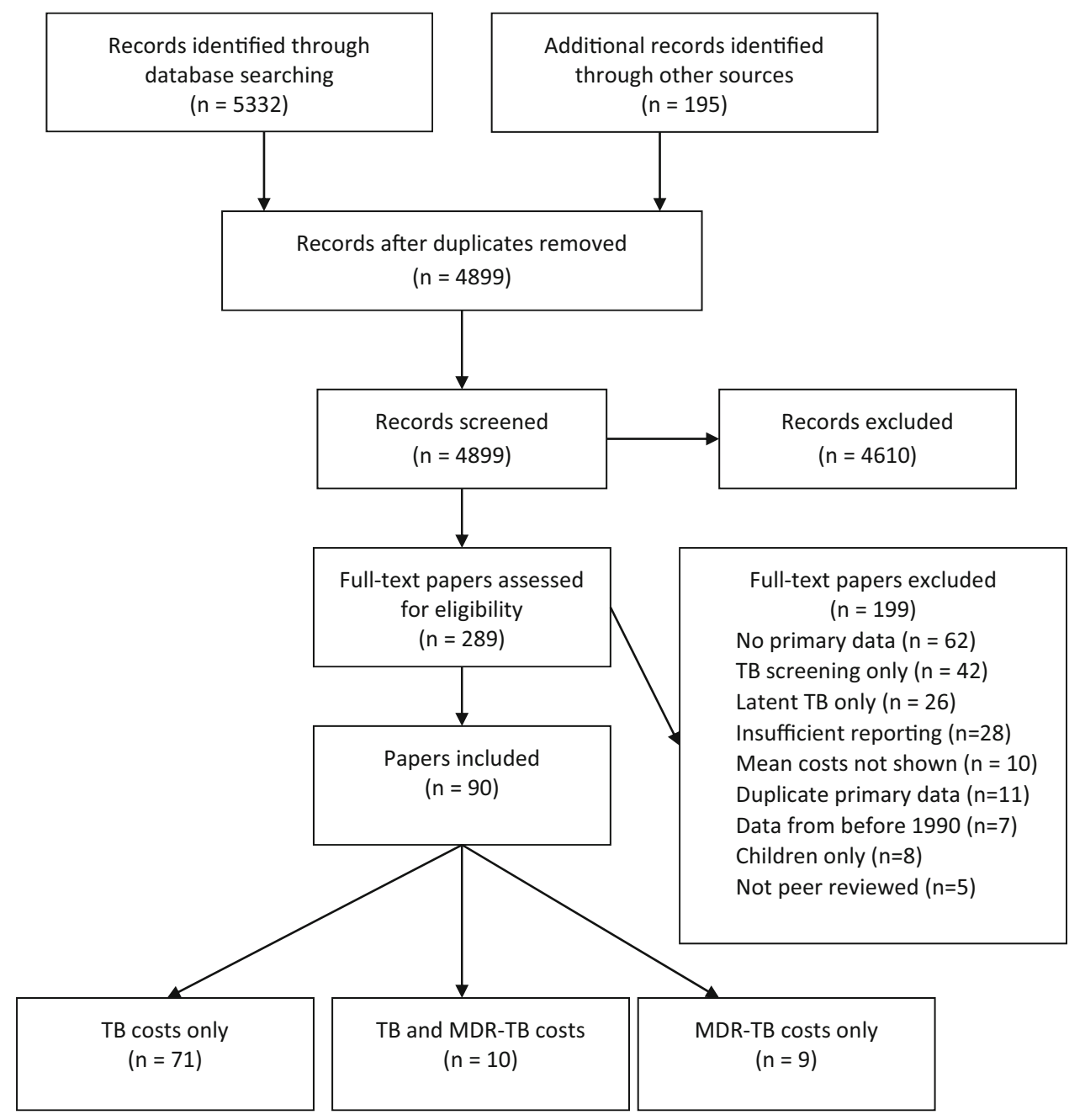

remaining $43 \%$ both provider and patient costs were evaluated (Table 1). This varied according to country income group. While a provider-only perspective was taken in 15 and 11 of the HIC and UMIC papers (60 and $38 \%$, respectively), only one LMIC paper (6\%) and one LIC paper $(5 \%)$ included provider costs only. Productivity losses were included in $81 \%$ of the papers that measured patient-incurred costs.

\subsection{Quality Assessment}

Quality assessment of individual papers is included in the ESM (Online Resources 2 and 3). Table 2 presents a summary according to country income group.

\subsubsection{Data Collection Methods}

The year of cost data and the main cost categories were adequately reported in $77 \%$ of papers (Table 2). In 79 of the 90 papers, cost data were collected from a sample of patients. The mean sample size across these studies were 324 patients (SD 532), ranging from nine in a MDR-TB study from the UK to 3510 patients in a German cost-ofillness study [23, 24]. However, in spite of relatively large sample sizes in many studies, only $30 \%$ presented descriptive statistics showing the spread around the mean cost values.

In ten of the remaining 11 studies, costs were determined by making assumptions about resources needed to treat TB according to national guidelines. For instance, a South African study by Pooran et al. [25] assumed that all DS-TB patients received drugs for 6 months and MDR-TB patients for 24 months, as this was the length of a full recommended treatment course. In the one study [26] that did not follow this approach, annual costs of primary health clinics in a specific area of South Africa were estimated and costs of TB treatment were determined by weighing total costs by the proportion of patients presenting due to TB [26]. 
Table 1 Summary of treatment cost papers included in review

\begin{tabular}{|c|c|c|c|c|c|c|}
\hline References & Year & Country & Interventions evaluated in study & $\begin{array}{l}\text { Provider } \\
\text { costs } \\
\text { included }\end{array}$ & $\begin{array}{l}\text { Direct } \\
\text { patient } \\
\text { costs } \\
\text { included }\end{array}$ & $\begin{array}{l}\text { Productivity } \\
\text { losses } \\
\text { included }\end{array}$ \\
\hline \multicolumn{7}{|l|}{ HICs $(n=25)$} \\
\hline Burman et al. [38] ${ }^{\mathrm{a}}$ & 1997 & USA & DOT vs. self-administered therapy & $\mathrm{X}$ & & $\mathrm{X}$ \\
\hline Palmer et al. [92] & 1998 & USA & Universal vs. partial DOT & $\mathrm{X}$ & & \\
\hline Migliori et al. [76] & 1998 & Russia & New vs. old treatment strategies & $\mathrm{X}$ & & \\
\hline Migliori et al. [78] & 1999 & Italy & DOT vs. DOT with staff incentives & $\mathrm{X}$ & & $\mathrm{X}$ \\
\hline Marchand et al. [93] & 1999 & Canada & Hospitalised treatment of elderly & $\mathrm{X}$ & & \\
\hline Weis et al. [33] & 1999 & USA & DOT vs. traditional therapy & $\mathrm{X}$ & & \\
\hline Wurtz and White [88] & 1999 & USA & Traditional therapy & $\mathrm{X}$ & & \\
\hline White and Moore-Gillon [23] ${ }^{\mathrm{a}}$ & 2000 & UK & Hospitalised treatment & $\mathrm{X}$ & & \\
\hline MacIntyre et al. [94] & 2001 & Australia & Inpatient vs. outpatient therapy & $\mathrm{X}$ & & \\
\hline Jacobs et al. [30] & 2002 & Russia & DOTS vs. traditional treatment & $\mathrm{X}$ & $\mathrm{X}$ & $\mathrm{X}$ \\
\hline Rajbhandary et al. $[28]^{\mathrm{b}}$ & 2004 & USA & MDR-TB & $\mathrm{X}$ & & $\mathrm{X}$ \\
\hline Atun et al. [40] & 2006 & Russia & TB control system & $\mathrm{X}$ & & \\
\hline Kang et al. $[31]^{\mathrm{b}}$ & 2006 & South Korea & MDR-TB & $\mathrm{X}$ & $\mathrm{X}$ & $\mathrm{X}$ \\
\hline Bocchino et al. [48] & 2006 & Italy & Integrated in- and outpatient & $\mathrm{X}$ & & \\
\hline Burns and Harrison [39] & 2007 & New Zealand & DOT in non-resident population & $X$ & & \\
\hline Kik et al. [29] & 2009 & Netherlands & Household costs of immigrants & & $\mathrm{X}$ & $\mathrm{X}$ \\
\hline Miller et al. [52] & 2010 & USA & Total TB costs in a Texas county & $\mathrm{X}$ & & $\mathrm{X}$ \\
\hline Montes-Santiago et al. [65] & 2010 & Spain & Hospitalisation only & $X$ & & \\
\hline Tu et al. [95] & 2011 & Taiwan & Comparison of diagnostic methods & $X$ & & \\
\hline Eralp et al. [51] & 2012 & UK & Screening, diagnosis and treatment & $X$ & & \\
\hline Diel et al. $[24]^{\mathrm{a}}$ & 2012 & Germany & Hospital and outpatient & $\mathrm{X}$ & & $\mathrm{X}$ \\
\hline Floyd et al. $[55]^{\mathrm{b}}$ & 2012 & Estonia, Russia & Traditional vs. WHO approach & $\mathrm{X}$ & & \\
\hline Miller et al. $[41]^{\mathrm{a}}$ & 2013 & Latvia & DOTS and MDR-TB & $\mathrm{X}$ & & \\
\hline Marks et al. $[56]^{\mathrm{b}}$ & 2014 & USA & Hospitalisation & $\mathrm{X}$ & & $\mathrm{X}$ \\
\hline Diel et al. $[87]^{\mathrm{b}}$ & 2014 & Germany & WHO guidelines & $\mathrm{X}$ & & $\mathrm{X}$ \\
\hline \multicolumn{7}{|l|}{ UMICs $(n=29)$} \\
\hline Masobe et al. [43] & 1995 & South Africa & Isoniazid prophylactic therapy & $\mathrm{X}$ & & \\
\hline Wilkinson et al. [49] & 1997 & South Africa & DOT vs. traditional treatment & $X$ & $\mathrm{X}$ & $\mathrm{X}$ \\
\hline Sawert et al. [77] & 1997 & Thailand & TB programme improvements & $X$ & & $\mathrm{X}$ \\
\hline Dick and Henchie [26] & 1998 & South Africa & TB programme in Cape Town & $X$ & & \\
\hline $\mathrm{Xu}$ et al. $[60]^{\mathrm{a}}$ & 2000 & China & DOTS vs. traditional treatment & $\mathrm{X}$ & & \\
\hline Suarez et al. $[62]^{\mathrm{b}}$ & 2002 & Peru & MDR-TB 2nd line drug treatment & $\mathrm{X}$ & & \\
\hline Kamolratanakul et al. $[61]^{\mathrm{a}}$ & 2002 & Thailand & Comparison of delivery centres & $\mathrm{X}$ & & \\
\hline Moalosi et al. [45] & 2003 & Botswana & Home-based vs. hospital DOT & $\mathrm{X}$ & $\mathrm{X}$ & $\mathrm{X}$ \\
\hline Ruiz et al. [96] & 2003 & Mexico & National costs & $\mathrm{X}$ & & \\
\hline Costa et al. $[81]^{\mathrm{a}}$ & 2005 & Brazil & Treatment in Salvador state & $\mathrm{X}$ & $\mathrm{X}$ & $\mathrm{X}$ \\
\hline Sinanovic and Kumaranayake [97] & 2006 & South Africa & Public-private partnership model & $\mathrm{X}$ & & \\
\hline Peralta Perez et al. [53] & 2006 & Cuba & DOTS & $\mathrm{X}$ & & \\
\hline Jackson et al. [98] & 2006 & China & Household costs & & $\mathrm{X}$ & $\mathrm{X}$ \\
\hline Liu et al. [99] & 2007 & China & Household costs & & $\mathrm{X}$ & \\
\hline Elamin et al. [72] & 2008 & Malaysia & Costs in Penang state & $\mathrm{X}$ & $\mathrm{X}$ & $\mathrm{X}$ \\
\hline Cusmano et al. [44] & 2009 & Argentina & DOTS & $\mathrm{X}$ & $\mathrm{X}$ & $\mathrm{X}$ \\
\hline Guzman-Montes et al. [100] & 2009 & Mexico & Household costs & & $\mathrm{X}$ & $\mathrm{X}$ \\
\hline Fairall et al. [42] & 2010 & South Africa & Educational outreach services & $X$ & $\mathrm{X}$ & \\
\hline
\end{tabular}


Table 1 continued

\begin{tabular}{|c|c|c|c|c|c|c|}
\hline References & Year & Country & Interventions evaluated in study & $\begin{array}{l}\text { Provider } \\
\text { costs } \\
\text { included }\end{array}$ & $\begin{array}{l}\text { Direct } \\
\text { patient } \\
\text { costs } \\
\text { included }\end{array}$ & $\begin{array}{l}\text { Productivity } \\
\text { losses } \\
\text { included }\end{array}$ \\
\hline Rouzier et al. $[86]^{\mathrm{a}}$ & 2010 & Ecuador & Household costs & & $\mathrm{X}$ & $\mathrm{X}$ \\
\hline Steffen et al. [14] & 2010 & Brazil & DOTS vs. non-DOTS & $X$ & $X$ & $X$ \\
\hline Prado et al. [80] & 2011 & Brazil & Guardians vs. heath workers & $X$ & $X$ & $X$ \\
\hline Samandari et al. $[101]^{\mathrm{a}}$ & 2011 & Botswana & DOTS for DS-TB and MDR-TB & $\mathrm{X}$ & & \\
\hline Nieto et al. [102] & 2012 & Colombia & Increased guardian supervision & $X$ & $X$ & \\
\hline Schnippel et al. [57] ${ }^{\mathrm{b}}$ & 2013 & South Africa & Hospitalised management & $\mathrm{X}$ & & \\
\hline Pooran et al. $[25]^{\mathrm{b}}$ & 2013 & South Africa & MDR-TB & $\mathrm{X}$ & & \\
\hline Zou et al. [103] & 2013 & China & DOTS incentives vs. no incentive & $X$ & $X$ & $X$ \\
\hline Pan et al. [63] & 2013 & China & DOTS & & $\mathrm{X}$ & $\mathrm{X}$ \\
\hline Wei et al. [104] & 2014 & China & DOTS & & $\mathrm{X}$ & \\
\hline Foster et al. [105] & 2015 & South Africa & DOTS & & $\mathrm{X}$ & $\mathrm{X}$ \\
\hline \multicolumn{7}{|l|}{ LMICs $(n=17)$} \\
\hline Rajeswari et al. [106] & 1999 & India & Household costs & & $\mathrm{X}$ & $\mathrm{X}$ \\
\hline Khan et al. [47] & 2002 & Pakistan & Health worker vs. family & $X$ & $\mathrm{X}$ & $\mathrm{X}$ \\
\hline Vassall et al. [46] & 2002 & Egypt, Syria & DOTS vs. previous strategies & $\mathrm{X}$ & $\mathrm{X}$ & $\mathrm{X}$ \\
\hline Nganda et al. [66] & 2003 & Kenya & Increased community involvement & $X$ & $X$ & $X$ \\
\hline Peabody et al. [107] & 2005 & Philippines & Economic burden of TB & $X$ & $X$ & \\
\hline Tupasi et al. [58] ${ }^{\mathrm{b}}$ & 2006 & Philippines & DOTS-Plus MDR-TB & $\mathrm{X}$ & $\mathrm{X}$ & \\
\hline Floyd et al. [34] & 2006 & India & Public-private mix DOTS & $X$ & $\mathrm{X}$ & $\mathrm{X}$ \\
\hline El-Sony et al. [54] & 2006 & Sudan & Comparison of HIV+ and HIV- & $X$ & & \\
\hline Aspler et al. [108] & 2008 & Zambia & Household costs & & $X$ & $X$ \\
\hline Muniyandi et al. [83] & 2008 & India & DOTS vs. non-DOTS & & $\mathrm{X}$ & $\mathrm{X}$ \\
\hline Pantoja et al. [32] & 2009 & India & Public-private mix DOTS & $X$ & $\mathrm{X}$ & $\mathrm{X}$ \\
\hline John et al. [82] & 2009 & India & DOTS & & $X$ & $\mathrm{X}$ \\
\hline Vassall et al. [35] & 2009 & Ukraine & DOTS implementation & $X$ & $\mathrm{X}$ & \\
\hline Mahendradhata et al. [79] & 2010 & Indonesia & Private practitioner referral & $\mathrm{X}$ & $\mathrm{X}$ & $\mathrm{X}$ \\
\hline Mauch et al. [109] & 2011 & Kenya & Household costs & & $X$ & $X$ \\
\hline Umar et al. [110] & 2012 & Nigeria & Household costs & & $X$ & \\
\hline Mauch [68] & 2013 & $\begin{array}{l}\text { Dom. Republic }{ }^{\mathrm{c}} \text {, } \\
\text { Ghana, Vietnam }\end{array}$ & Household costs & & $\mathrm{X}$ & $\mathrm{X}$ \\
\hline \multicolumn{7}{|l|}{ LICs $(n=19)$} \\
\hline Saunderson $[50]$ & 1995 & Uganda & Hospital vs. ambulatory care & $X$ & $\mathrm{X}$ & $\mathrm{X}$ \\
\hline Maponga et al. [89] & 1996 & Zimbabwe & TB/HIV co-epidemic & $X$ & & \\
\hline Gibson et al. [111] & 1998 & Sierra Leone & Household costs & & $\mathrm{X}$ & \\
\hline Wyss et al. [67] & 2001 & Tanzania & Household costs & & $\mathrm{X}$ & $\mathrm{X}$ \\
\hline Islam et al. [73] & 2002 & Bangladesh & CHW vs. no $\mathrm{CHW}$ & $\mathrm{X}$ & $X$ & $\mathrm{X}$ \\
\hline Floyd et al. [36] & 2003 & Malawi & Increased community involvement & $X$ & $X$ & $X$ \\
\hline Okello et al. [37] & 2003 & Uganda & Increased community involvement & $X$ & $X$ & $\mathrm{X}$ \\
\hline Wandwalo et al. [85] & 2005 & Tanzania & Community vs. health facility & $\mathrm{X}$ & $\mathrm{X}$ & $\mathrm{X}$ \\
\hline Jacquet et al. [64] & 2006 & Haiti & DOTS expansion & $X$ & $X$ & $\mathrm{X}$ \\
\hline Karki et al. [112] & 2007 & Nepal & Public-private partnership & $X$ & $X$ & $\mathrm{X}$ \\
\hline Mirzoev et al. [113] & 2008 & Nepal & Community vs. family observation & $X$ & $\mathrm{X}$ & $\mathrm{X}$ \\
\hline Aye et al. [69] & 2010 & Tajikistan & Household costs & & $X$ & $\mathrm{X}$ \\
\hline Datiko et al. [84] & 2010 & Ethiopia & Health extension workers & $X$ & $X$ & $X$ \\
\hline Vassall et al. [74] & 2010 & Ethiopia & Collaborative TB-HIV & & $\mathrm{X}$ & $\mathrm{X}$ \\
\hline
\end{tabular}


Table 1 continued

\begin{tabular}{|c|c|c|c|c|c|c|}
\hline References & Year & Country & Interventions evaluated in study & $\begin{array}{l}\text { Provider } \\
\text { costs } \\
\text { included }\end{array}$ & $\begin{array}{l}\text { Direct } \\
\text { patient } \\
\text { costs } \\
\text { included }\end{array}$ & $\begin{array}{l}\text { Productivity } \\
\text { losses } \\
\text { included }\end{array}$ \\
\hline Pichenda et al. [59] ${ }^{\mathrm{a}}$ & 2012 & Cambodia & Early diagnosis and non-hospital & $\mathrm{X}$ & $\mathrm{X}$ & $\mathrm{X}$ \\
\hline Laokri et al. [114] & 2013 & Burkina Faso & Household costs & & $X$ & \\
\hline Yitayal et al. [71] & 2014 & Ethiopia & DOTS & & $\mathrm{X}$ & $\mathrm{X}$ \\
\hline Laokri et al. [115] & 2014 & Benin & DOTS & & $X$ & \\
\hline $\begin{array}{l}\text { Gospodarevskaya } \\
\text { et al. }[75]\end{array}$ & 2014 & Bangladesh, Tanzania & $\begin{array}{l}\text { DOT female community } \\
\text { worker; DOT family }\end{array}$ & & $X$ & $\mathrm{X}$ \\
\hline
\end{tabular}

$\mathrm{X}$ indicates the category of cost (Provider, Direct patient, or Productivity losses)

$C H W$ community health worker, DOT directly observed treatment, DOTS directly observed treatment-short course, DS-TB drug-susceptible tuberculosis, HIC high-income country, LIC low-income country, LMIC lower-middle income country, MDR-TB multidrug-resistant tuberculosis, UMIC upper-middle income country, WHO World Health Organization

${ }^{a}$ Both DS-TB and MDR-TB costs

b MDR-TB costs only

${ }^{c}$ Dominican Republic is an upper-middle income country

Table 2 Quality assessment: percent of papers ${ }^{\mathrm{a}, \mathrm{b}}$

\begin{tabular}{|c|c|c|c|c|c|c|c|c|c|}
\hline & $\begin{array}{l}\text { Mean } \\
\text { number of } \\
\text { patients in } \\
\text { study } \\
\text { sample }^{c}\end{array}$ & $\begin{array}{l}\text { Ingredient } \\
\text { approach } \\
\text { used for } \\
\text { provider } \\
\text { costs }\end{array}$ & $\begin{array}{l}\text { Resource use } \\
\text { and unit } \\
\text { costs clearly } \\
\text { described }\end{array}$ & $\begin{array}{l}\text { Year of } \\
\text { cost } \\
\text { data } \\
\text { reported }\end{array}$ & $\begin{array}{l}\text { Main cost } \\
\text { categories } \\
\text { clearly } \\
\text { separated }\end{array}$ & $\begin{array}{l}\text { Descriptive } \\
\text { statistics } \\
\text { presented }\end{array}$ & $\begin{array}{l}\text { Patient } \\
\text { interviews }\end{array}$ & $\begin{array}{l}\text { Methods for } \\
\text { valuing } \\
\text { productivity } \\
\text { loss clearly } \\
\text { explained }\end{array}$ & $\begin{array}{l}\text { Sources for } \\
\text { productivity } \\
\text { losses } \\
\text { assumptions } \\
\text { justified }\end{array}$ \\
\hline \multicolumn{10}{|c|}{ Papers with provider costs only $(n=28)$} \\
\hline $\begin{array}{l}\mathrm{HIC} \\
(n=15)\end{array}$ & 307 & 73 & 60 & 80 & 73 & 20 & NA & NA & NA \\
\hline $\begin{array}{l}\text { UMIC } \\
(n=11)\end{array}$ & 384 & 100 & 73 & 91 & 82 & 9 & NA & NA & NA \\
\hline $\begin{array}{l}\text { LMIC } \\
(n=1)\end{array}$ & 1797 & 0 & 0 & 0 & 100 & 0 & NA & NA & NA \\
\hline $\begin{array}{l}\text { LIC } \\
(n=1)\end{array}$ & 300 & 100 & 0 & 100 & 0 & 0 & NA & NA & NA \\
\hline \multicolumn{10}{|c|}{ Papers with patient costs included $(n=62)$} \\
\hline $\begin{array}{l}\text { HIC } \\
(n=10)\end{array}$ & 475 & 89 & 82 & 82 & 82 & 45 & 18 & 82 & 64 \\
\hline $\begin{array}{l}\text { UMIC } \\
(n=18)\end{array}$ & 305 & 91 & 78 & 61 & 83 & 22 & 94 & 61 & 56 \\
\hline $\begin{array}{l}\text { LMIC } \\
(n=16)\end{array}$ & 345 & 63 & 94 & 88 & 81 & 50 & 94 & 69 & 50 \\
\hline $\begin{array}{l}\text { LIC } \\
(n=18)\end{array}$ & 154 & 73 & 78 & 78 & 89 & 33 & 100 & 56 & 44 \\
\hline All papers & 324 & 81 & 76 & 77 & 80 & 30 & 83 & 65 & 52 \\
\hline
\end{tabular}

HIC high-income countries, LIC low-income country, LMIC lower-middle income country, NA not applicable, UMIC upper-middle income countries

${ }^{a}$ These results are shown for each study in the Electronic Supplementary Material (Online Resources 2 and 3)

${ }^{\mathrm{b}}$ Data are presented as \% unless otherwise indicated

c Among the studies with patient-level data 


\subsubsection{Provider Costs}

The ingredient approach, which entails determining resource quantities and unit costs separately, is generally viewed as the most robust and transparent method for provider cost estimation [27]. This approach was transparently used in 54 of the 67 studies that included provider costs. While the method may also have been partly used in the remaining 13 studies, techniques were not clearly described, and resource quantities and unit costs were not separately presented in these papers.

\subsubsection{Patient-Incurred Costs}

Patient interviews were conducted in 52 of the 62 studies that included a patient perspective. The mean sample size was 298 (SD 527) patient interviews, ranging from 13 patients in a US study to 3510 in the German cost-of-illness study [24, 28]. Patient interviews were more common in LICs and middle-income countries than in HICs. While ten studies from HICs included patient-incurred costs, interviews were only conducted in two of these, a study from
Holland assessing costs among immigrant TB patients and an economic evaluation of the Russian TB treatment scheme and short-course chemotherapy [29, 30]. In the other eight studies, productivity losses (and transport costs in a study from South Korea [31]) were the only type of patient costs included and these were estimated without data from interviews.

Methods used for estimating productivity losses varied in six different ways in the 51 papers that included these: (1) patients were interviewed about their loss of income ( $n=15)$; (2) patients were interviewed about productive time lost and on their income before falling ill; productivity losses were then calculated by multiplying mean income across the patient sample with reported time loss $(n=12)$; (3) patients were interviewed about productive time lost, which was multiplied by an official wage rate $(n=11)$; (4) assumptions were made about the length of time patients were not able to work, which was valued using an official wage rate $(n=7)$; (5) a value was placed on death based on average lifetime income or GNI per capita, which was multiplied by estimated life-years lost $(n=4)$; and (6) methods were not clear $(n=2)$. Across the 51 studies, only

Table 3 Mean drug-sensitive and multidrug-resistant tuberculosis provider treatment costs according to country income group ${ }^{\mathrm{a}}$

\begin{tabular}{|c|c|c|c|c|c|c|c|}
\hline Income group & Hospitalisation & Outpatient visits & Drugs & $\begin{array}{l}\text { Diagnostic and } \\
\text { monitoring tests }\end{array}$ & Other $^{\mathrm{b}}$ & Total $^{\mathrm{c}}$ & $\mathrm{SD}^{\mathrm{d}}$ \\
\hline \multicolumn{8}{|l|}{ DS-TB } \\
\hline $\mathrm{HIC}(n=19)$ & $11,283(8)$ & $1471(5)$ & $1392(6)$ & $961(7)$ & $3413(5)$ & $14,659(19)$ & 13,594 \\
\hline $\mathrm{UMIC}(n=19)$ & $380(5)$ & $218(10)$ & $107(14)$ & $69(11)$ & $386(9)$ & $840(19)$ & 1105 \\
\hline LMIC $(n=10)$ & $215(4)$ & $75(6)$ & $39(6)$ & $48(8)$ & $25(5)$ & $273(10)$ & 212 \\
\hline $\operatorname{LIC}(n=11)$ & $128(2)$ & $61(5)$ & $49(8)$ & $19(3)$ & $50(8)$ & $258(11)$ & 352 \\
\hline All income groups $\left(\right.$ papers $\left.=58^{\mathrm{e}}\right)$ & 4909 (19) & $396(26)$ & $329(32)$ & $453(26)$ & $744(27)$ & $6667(59)$ & 10,105 \\
\hline Proportion, $\%$ & 73.6 & 5.9 & 4.6 & 4.1 & 11.7 & 99.9 & \\
\hline \multicolumn{8}{|l|}{ MDR-TB } \\
\hline $\mathrm{HIC}(n=10)$ & $53,078(10)$ & $18,720(7)$ & $19,887(8)$ & $1201(6)$ & $1841(3)$ & $83,365(10)$ & 64,825 \\
\hline UMIC $(n=7)$ & $6056(2)$ & $622(3)$ & $2052(6)$ & $350(5)$ & $823(5)$ & $5284(7)$ & 3420 \\
\hline LMIC $(n=1)$ & $207(1)$ & $218(1)$ & $2930(1)$ & $397(1)$ & $52,567(1)$ & $6313(1)$ & NA \\
\hline LIC $(n=1)$ & NI & NI & NI & NI & NI & $1218(1)$ & NA \\
\hline All income groups $\left(\right.$ papers $\left.=18^{f}\right)$ & $41,776(13)$ & $12,102(11)$ & $11,623(15)$ & $779(12)$ & $1356(9)$ & $46219(19)$ & 61,027 \\
\hline Proportion, $\%$ & 61.8 & 17.9 & 17.2 & 1.2 & 2.0 & 100.1 & \\
\hline
\end{tabular}

Data are presented as US\$, year 2014 values (number) unless otherwise indicated

$D S$ drug susceptible, HIC high-income country, LIC low-income country, LMIC lower-middle income country, MDR multidrug-resistant, NA not applicable, $N I$ cost not itemised, TB tuberculosis, UMIC upper-middle income country

a These are shown for each study in the Electronic Supplementary Material (Online Resources 4-7)

b Other provider costs include start-up costs, treatment supervision, staff salary and training, advocacy, adverse effects, contact tracing, supplies and transportation; or in some papers, where costs were not disaggregated, the total treatment costs to the provider, including supervision, training, supplies and drugs

c Total $\neq$ sum of categories because some papers did not itemise costs and only reported total costs

${ }^{d}$ Vassall et al. [46] (2002) presented two LMIC studies (Egypt and Syria) in one paper

e Floyd et al. [55] (2012) presented two HIC studies (Estonia and Russia) in one paper

${ }^{\mathrm{f}}$ Standard deviation for total mean provider treatment costs 
Table 4 Mean drug-sensitive and multidrug-resistant tuberculosis direct patient costs and productivity losses according to country income group $^{\mathrm{a}}$

\begin{tabular}{|c|c|c|c|c|c|c|c|c|}
\hline Income group & $\begin{array}{l}\text { Clinic visits and } \\
\text { clinical tests } \\
\text { user fees }\end{array}$ & Drugs & Transport & Other $^{\mathrm{b}}$ & $\begin{array}{l}\text { Total direct } \\
\text { costs }^{\mathrm{c}}\end{array}$ & $\mathrm{SD}^{\mathrm{d}}$ & $\begin{array}{l}\text { Productivity } \\
\text { losses }\end{array}$ & $\mathrm{SD}^{\mathrm{e}}$ \\
\hline \multicolumn{9}{|l|}{ DS-TB } \\
\hline $\mathrm{HIC}(n=6)$ & $107(1)$ & NI & $260(1)$ & $379(1)$ & $373(2)$ & 106 & $2801(6)$ & 2018 \\
\hline UMIC $(n=19)$ & $221(9)$ & $62(4)$ & $120(13)$ & $491(12)$ & $603(18)$ & 868 & $600(12)$ & 847 \\
\hline LMIC $(n=17)$ & $55(9)$ & $21(7)$ & $9(4)$ & $47(10)$ & $84(17)$ & 90 & $238(11)$ & 320 \\
\hline LIC $(n=19)$ & $49(13)$ & $38(5)$ & $45(10)$ & $96(16)$ & $155(19)$ & 164 & $248(14)$ & 266 \\
\hline All income groups $\left(\right.$ papers $=53^{\mathrm{f}, \mathrm{g}, \mathrm{h}}$ ) & $101(32)$ & $36(16)$ & $82(28)$ & $212(39)$ & $432(36)$ & 544 & $700(43)$ & 1229 \\
\hline Proportion, \% & 23.3 & 8.5 & 19.1 & 49.1 & 100.0 & & & \\
\hline \multicolumn{9}{|l|}{ MDR-TB } \\
\hline $\mathrm{HIC}(n=5)$ & $\mathrm{CNI}$ & $\mathrm{CNI}$ & $21(1)$ & $\mathrm{CNI}$ & $21(1)$ & NA & $49,204(5)$ & 51,216 \\
\hline UMIC $(n=2)$ & $12(2)$ & NI & $178(2)$ & $470(2)$ & $660(2)$ & 394 & $3532(2)$ & 4578 \\
\hline $\operatorname{LMIC}(n=1)$ & $909(1)$ & NI & NI & $707(1)$ & $1616(1)$ & NA & $\mathrm{CNI}$ & NA \\
\hline LIC $(n=1)$ & $103(1)$ & NI & $18(1)$ & $285(1)$ & $406(1)$ & NA & $1256(1)$ & NA \\
\hline All income groups $($ papers $=9$ ) & $259(4)$ & NI & $99(4)$ & $483(4)$ & $672(5)$ & 621 & $28,260(8)$ & 45,605 \\
\hline Proportion, \% & 30.8 & 0.0 & 11.7 & 57.4 & 99.9 & & & \\
\hline
\end{tabular}

Data are presented as US\$, year 2014 values (number) unless otherwise indicated

$C N I$ cost not included, DS drug susceptible, HIC high-income country, LIC low-income country, LMIC lower-middle income country, MDR multidrug-resistant, $N A$ not applicable, $N I$ cost not itemised, $S D$ standard deviation, $T B$ tuberculosis, UMIC upper-middle income country

${ }^{a}$ These are shown for each paper in the Electronic Supplementary Material (Online Resources 4-7)

${ }^{\mathrm{b}}$ Other patient costs typically include direct medical costs (non-TB drugs, hospitalisation) and direct non-medical costs (food, drink, vitamins, traditional medicine, and accommodation), or in some papers, where costs were not disaggregated, the total costs during pre-diagnosis, diagnosis, intensive treatment and continuation treatment phases

c Total $\neq$ sum of categories because some papers did not itemise costs and only reported total costs

${ }^{d}$ Mauch et al. [68] (2013) presented one UMIC study (Dominican Republic) and two LMIC studies (Ghana and Vietnam) in one paper

e Vassall et al. [46] (2002) presented two LMIC studies (Egypt and Syria) in one paper

${ }^{\mathrm{f}}$ Gospodarevskya et al. [75] (2014) presented two LIC studies (Bangladesh and Tanzania) in one paper

g SD for total mean patient costs

${ }^{\mathrm{h}} \mathrm{SD}$ for mean productivity losses

$65 \%$ clearly explained the methods used for productivity losses, and $52 \%$ justified the sources used for these estimates.

\subsection{Mean Costs Per Patient}

Mean provider and patient-incurred costs per patient are summarised in Tables 3 and 4 according to country income groups. These data are presented for each study in the ESM (Online Resources 4-7).

\subsubsection{Drug-Susceptible (DS) Tuberculosis (TB) Provider Costs}

DS-TB provider costs were positively correlated with GNI per capita $(r=0.73, p<0.001)$. A scatterplot illustrates the relationship (Fig. 2). Mean DS-TB treatment costs per patient were 57 times higher in HICs [US $\$ 14,659$ (SD 13,594)] than in LICs [US\$258 (SD 352)]. A high degree of variability was observed in income group cost values, with the SD being almost as large as the mean provider costs in HICs and larger for LICs.

3.4.1.1 Hospitalisation and Outpatient Care Across all 59 studies, hospitalisation accounted for $74 \%$ of all DSTB provider costs (Table 3). Hospitalisation accounted for $63 \%$ in HICs (US\$11,283), $51 \%$ in LMICs (US\$215) and LICs (US\$128), but only $12 \%$ in UMICs (US\$380). However, within the income groups, the proportion of hospitalisation costs varied widely between studies, with $2 \%$ for an unreported number of hospital days in a publicprivate sector implementation scenario in India to $81 \%$ in a study on DOT in Texas, USA, with 23 hospital days [32, 33]. Among LMICs, India consistently had the lowest costs for hospitalisation and the other cost categories [32, 34]. In LMIC costs, Ukraine had the highest hospitalisation and outpatient costs, at approximately twice the average income group costs [35]. Only two of the 11 LIC studies 


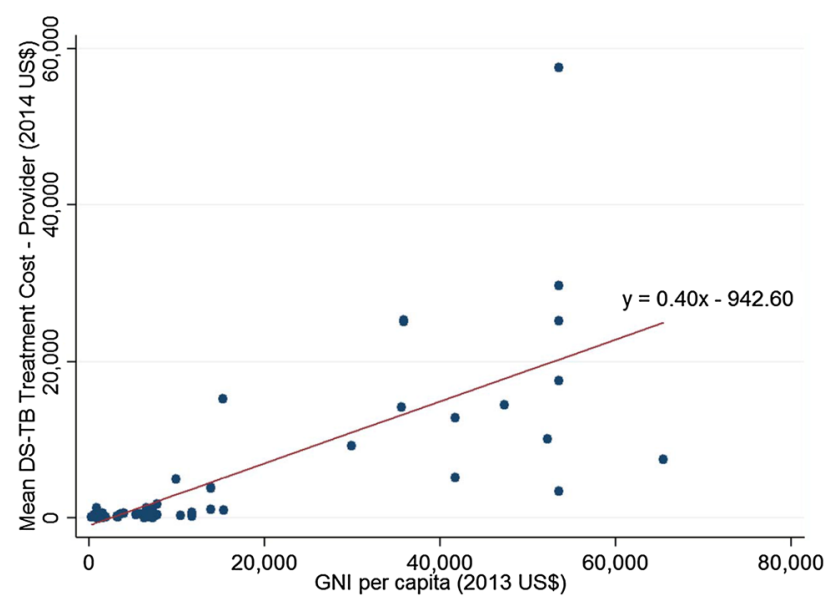

Fig. 2 Mean tuberculosis provider treatment costs per patient (US\$, year 2014 values) according to GNI per capita (\$US, year 2013 values)

reported hospitalisation costs, with US\$75 (60\%) in Malawi [36], and US\$181 (50\%) in Uganda [37].

Mean outpatient treatment costs were 12 times less than hospitalisation costs and accounted for only $6 \%$ of total costs. However, the importance of outpatient costs varied substantially among country income groups. In HICs, only five of 19 studies reported any outpatient costs [24, 38-41]. Of the ten UMIC studies that reported these costs, Argentina and South Africa had the lowest values of around US\$20 per patient [42-44] and Botswana the highest at US\$658 per patient [45]. Egypt was an outlier among the LMICs, reporting outpatient costs of US\$187 [46], which was 15-25 times more than in Pakistan (US\$11) and India (US\$6) [32, 47]. In LICs, five studies reported outpatient costs, with a mean of US\$61.

3.4.1.2 Drugs Costs of DS-TB drugs were, on average, $5 \%$ of total costs, but varied widely between settings, from a mean of US\$49 across LICs to US\$1392 in HICs. Within the HICs, drug costs were US\$311 in a US study [38], US\$654 in another US study [33], and as much as US\$4055 for an unstated combination of DS-TB and MDR-TB drugs in Italy [48]. Within this group of countries, it is difficult to discern whether drug costs have decreased or increased over time because only six of the 19 studies presented disaggregated drug costs. In upper middle-income South Africa, drug costs appear to have decreased from US\$46 in 1994 [49] to US\$3 in 2003 [42]. In LMICs and LICs, drug costs were lowest in India at approximately US $\$ 15$ between 2002 and 2005 and highest in Uganda at US\$166 in 1992 [32, 34, 50]. Without these outliers, mean drug costs in LMICs and LICs were US\$51 and US\$33, respectively, with data from between 1992 and 2007.
3.4.1.3 Diagnostics and Monitoring Tests Unlike the costs of drugs and hospitalisation, mean costs per patient for diagnostics and monitoring tests were relatively similar across income groups. In the UK, costs of TB tests in a population of healthcare workers were US $\$ 157$ [51]. In three US studies on urban DOT programmes by Miller et al. [52], Burman et al. [38] and Weis et al. [33], costs per patient were reported as US\$124, US\$635 and US\$1505, respectively. In the Miller et al. [52] study, only one acidfast bacilli (AFB) smear and culture was included, while the other studies typically included at least two chest $\mathrm{X}$-rays, four sputum cultures and one tuberculin skin test (TST). Burman et al. [38] additionally included five serum bilirubin tests and five aspartate aminotransferase tests. Interferon-Gamma Release Assay was used instead of sputum culture in the UK study by Eralp et al. [51]. Although this review excluded latent TB, some studies presented aggregated costs for diagnosis of latent TB together with tests for diagnosis and monitoring of active TB, in which case the cost of the latent TB tests could not be excluded.

Mean diagnostics and monitoring costs in UMICs were US\$69, with Cuba being the only outlier at US\$289 in 2002 [53]. A Sudanese study that compared the costs of managing HIV-positive and HIV-negative TB patients reported the largest monitoring costs within this income group at US\$135 per patient [54].

\subsubsection{Multidrug-Resistant-TB (MDR-TB) Provider Costs}

Mean provider costs for MDR-TB treatment were US $\$ 83,365$ (SD 64,835) for the ten included studies and far less for the seven UMICs at US\$5284 (SD 3420). A large variation in costs was observed for both groups; measures of spread were not available for the LMIC and LIC groups as there was only one study included in each of these categories.

\subsubsection{Hospitalisation and Outpatient Care MDR-TB} hospitalisation represented the highest proportion of provider costs in HICs, contributing to $64 \%$ (Table 3). Even though patients were hospitalised for an average of 192 days in Estonia, hospitalisation represented only $50 \%$ of total costs at US\$8007 [55]. In the USA Burman et al. [38] reported hospitalisation costs four times higher than those reported by Rajbhandary et al. [28] because the length of stay decreased from an average of 90 days in 1994 to 28 days in 2000 (US $\$ 181,909$ vs. 41,612 ) $[28,38]$. The mean length of MDR-TB hospitalisation in the USA in 2010, as reported by Marks et al. [56], remained 28 days, but the cost, US $\$ 87,619$, more than doubled the 2000 value calculated by Rajbhandary et al. [28]. 
In Russia, hospitalisation and outpatient care accounted for 37 and $3 \%$, respectively, if treatment is delivered according to WHO guidelines [55]. This Russian study reported an average of 321 days in hospital, costing US\$6493, which was the second lowest value in the HIC group. In South Korea, hospitalisation costs were the least per patient (US\$3521), but the length of stay was only 8 days, by far the shortest stay for any HIC or UMIC [31].

In South Africa, Schnippel et al. [57] reported a mean hospitalisation period of 105 days, contrasting greatly with Pooran et al. [25], who estimated costs according to prevailing guidelines, which recommended complete outpatient care for smear-negative MDR-TB patients [25, 57]. Pooran et al. [25] estimated that surgery, which we presented as a hospital cost, amounted to US\$97 (2\%) per patient, while outpatient visits totalled US\$712 (17\%). Schnippel et al. [57] reported no outpatient costs, but hospitalisation accounted for $95 \%$ of total MDR-TB costs (US\$12,666). Both treatment scenarios are present in South Africa, but only $10 \%$ of MDR-TB patients are thought to require hospitalisation [25].

Only the Philippines were represented in the LMIC group. In a DOT short-course plus pilot programme, hospitalisation for 7 days amounted to $3 \%$ and outpatient visits to $4 \%$ of total costs [58]. In the LIC group, no disaggregated data were included for the one country represented, Cambodia, but the total MDR-TB cost was the lowest value of any country at US\$1218 [59].

3.4.2.2 Drugs In Estonia, 18 months of first- and secondline drugs amounted to half of the hospitalisation costs (192 hospital days) [55] (ESM Annex 5). The 2004 US study by Rajbhandary et al. [28] did not separately report costs of drugs, tests and personnel, but the 1997 US study by Burman et al. [38] calculated MDR-TB drug costs as amounting to approximately US\$12,000 per patient (6\%) $[28,38]$.

\subsubsection{Diagnostic and Monitoring Tests Mean costs per} MDR-TB patient for diagnostics and monitoring tests were US\$779 (1\%) across the 12 studies reporting these data. This accounted for less than $1 \%$ of total treatment costs in both the USA and the UK [23, 38]. In the South Korea study, diagnostics and monitoring test amounted to $24 \%$ of total costs [31]. The costs reported in the UK were for drug monitoring only, while Estonia, Germany, South Korea and the USA (Burman et al. [38]) each included at least one drug susceptibility test, 13 sputum culture tests and a combination of audiograms (USA), sputum smear tests (Estonia), X-rays, liver function and blood count tests.

The mean costs of diagnostics and monitoring tests were US\$350 in UMICs, ranging from US\$82 in China to US\$1013 in South Africa [25, 60]. With the exception of the South African study by Schnippel et al. [57], all studies reported at least eight sputum smear tests. Costs of sputum culture tests were included in all studies, with for instance three tests per patient in 24 months in Thailand and 18 tests per patient in 18 months in Peru [61, 62]. Costs of at least four chest X-rays per patient were included in all UMIC studies, except in South Africa where only one chest X-ray was included and all patients were hospitalised during the intensive phase of treatment [57]. Drug-susceptibility tests were reported in Thailand and in the two studies from South Africa [25, 57, 61]. In the only LMIC, the Philippines, costs of 34 smear tests, 27 culture tests, two drugsusceptibility tests and three X-rays were estimated at US\$397 per patient, equivalent to $6 \%$ of total costs [58].

\subsubsection{DS-TB Patient Costs}

Across all 61 studies, in 57 papers, mean direct costs incurred by patients was US\$432 (SD 544), ranging from US\$4 in Egypt to US\$3525 in China (Table 4) [46, 63]. Approximately half of patient costs, the highest proportion, was recorded in the 'other' category, which mainly consisted of non-TB drugs and food while hospitalised, or aggregated direct (medical and/or non-medical) patient costs. User fees comprised $23 \%$, drugs $9 \%$, and transportation $19 \%$ of total costs. In contrast to provider costs, there was no clear relationship between patient-incurred costs and GNI per capita. UMIC studies reported the highest mean patient costs (US\$603), followed by two HIC studies (US\$373), LICs (US\$155), and LMICs (US\$84).

3.4.3.1 User Fees The user fees category comprised costs incurred by patients for medical consultations or examinations when attending clinics or other health facilities during treatment, or for diagnostic or monitoring tests. Mean user fees payments were similar in high-income Netherlands and upper-middle income Botswana at around US\$105. In the Netherlands, user fees accounted for $22 \%$ of patient payments, but only for $11 \%$ in Botswana [29, 45].

Patients in upper-middle income Mexico and low-income Haiti paid the highest user fees of US\$344 and US\$299, respectively [64, 65]. The greatest proportion of total direct costs spent on user fees were in India (80\%), Kenya (69\%), South Africa (68\%) and Tanzania (66\%) [34, 42, 66, 67]. In general, user fees appeared to constitute the greatest proportion of patient costs in LMICs and smallest in UMICs.

3.4.3.2 Drugs No out-of-pocket payments were paid for drugs in HICs. Patients in Vietnam paid the least for drugs (US\$1), followed by the patients in the Dominican Republic (US\$5) [68]. Studies in Tajikistan and China 
reported the highest payments of US\$126 and US\$118, respectively $[69,70]$. In most LMICs, drug expenses were around US\$20, ranging between US\$1 and US\$63. Only five studies reported payments for drugs in LICs, ranging from US\$4 in Ethiopia to US\$126 in Tajikistan [69, 71].

3.4.3.3 Transportation The only HIC study that reported transportation costs was in Russia, with US\$260 [30]. Transportation costs in UMICs accounted for $1 \%$ of direct costs in the Dominican Republic and as much as $85 \%$ in Malaysia [68, 72]. In LMICs, patients paid an average of US\$9, accounting for between 2 and $28 \%$ of total direct costs. Ten of the 19 LIC studies reported transportation costs, accounting for $80 \%$ of direct costs in Bangladesh and between 22 and $44 \%$ in Ethiopia, Tajikistan and Tanzania [67, 69, 71, 73-75].

3.4.3.4 Productivity Losses Time lost due to seeking treatment and being ill with DS-TB was reported as 81 days in the Netherlands, 60 days in Thailand, 30 days in Italy, 25 days in the USA, 14 days in Malaysia, and $50 \%$ disabled for 2 months in Haiti [29, 64, 72, 76, 77].

DS-TB productivity losses increased with increasing GNI per capita, but amounts varied widely within country income groups (SD 1229) with a mean loss of US\$700 per patient for 43 studies. In HICs, values varied from US $\$ 450$ in Russia to US\$6246 in Italy [30, 78]. In UMICs, the range was between US $\$ 46$ in Argentina and US\$3048 in China [44, 63]. In LMICs, an Indonesian study reported productivity losses of US\$12 per patient compared with US\$996 in Vietnam [68, 79]. In LICs, costs were US\$11 in Bangladesh versus US\$775 in Tajikistan $[69,73]$. Studies from similar countries also showed quite different productivity loss estimates, such as US\$11 and US\$332 in Brazil [80, 81], US\$52 and US\$636 in India [82, 83], US\$9 and US\$200 in Ethiopia [71, 84], and US\$18 and US\$825 in Tanzania [67, 85].

\subsubsection{MDR-TB Patient Costs}

Mean direct costs incurred by MDR-TB patients were US\$672 (SD 621) across five studies (Table 4). The 'other' category constituted $57 \%$ of total costs, which included food, non-TB medication, follow-up tests and ventilation improvements to family homes. No patient costs were reported for MDR-TB drugs in any studies.

3.4.4.1 User Fees Similar to DS-TB user fees, MDR-TB user fees incurred by patients were for medical consultations or examinations at health facilities or to obtain diagnostic or monitoring tests. The mean MDR-TB user fees were US\$259, which were almost three times higher than for DS-TB. The Philippines reported the highest user fees at US\$909, accounting for $56 \%$ of all direct MDR-TB patient costs [58]. The user fees in low-income Cambodia (US\$103) were around eight times more than in UMICs (US\$4-20) [59]. No user fees were reported in HICs.

3.4.4.2 Transportation Mean transport costs incurred from receiving DS-TB treatment were US $\$ 99$ across the four studies. The highest costs were reported in the two UMICs, Brazil and Ecuador, at US\$90 and US\$266, respectively $[81,86]$. Studies from high-income South Korea and low-income Cambodia each reported around US $\$ 20$ per patient $[31,59]$.

3.4.4.3 Productivity Losses Productivity loss amounted to US $\$ 28,260$ per patient across the eight studies that included these values. As with DS-TB, productivity losses increased with income group, ranging from US\$295 in Brazil to US $\$ 136,802$ in the USA and an overall SD of US $\$ 45,605[56,81]$. Only three studies, two from the USA and one from Germany, clearly stated productive time lost for MDR-TB, which was 6, 24 and 8 months, respectively $[38,56,87]$.

\section{Discussion}

Costs of TB treatment vary substantially globally, with LICs adopting comparatively low-cost ambulatory methods of treatment delivery and benefitting from lower drug regimen prices than HICs. Provider costs are strongly correlated with GNI per capita. The cost of treating DS-TB from the provider perspective ranged from US $\$ 45$ in Zimbabwe to US $\$ 57,559$ in one of the US studies $[88,89]$. Provider costs of MDR-TB treatment are substantially higher than those for DS-TB and varied from US\$1218 in Cambodia to US $\$ 204,876$ in the USA $[38,59]$. It should be noted that many MDR-TB patients are primarily infected with transmitted MDR-TB strains and do not acquire the disease through misuse of their first-line regimen, so on the individual level these are not always alternative treatments. However, at a population level, the origins of MDR-TB lie in the misuse of TB drugs, and the improved delivery of first-line treatment can potentially reduce the level of comparatively high MDR-TB treatment costs $[90,91]$.

We also observed substantial cost variation within country income level groups. In the case of DS-TB, very low comparative costs were observed in LICs and LMICs using community-based models of care. While this suggests that devolvement of TB treatment at the community level may be efficient, the cost of community provision of TB treatment depends strongly on whether community workers are paid, and the valuation of their time. In the case of MDR-TB treatment, the variation in costs within country income level groups was also substantial. Lower 
cost treatment within groups was observed where primarily ambulatory models of care are adopted, with hospitalisation being a major driver of total cost. Across DS-TB treatment, there is a clear reduction in costs over time due to the extent of hospitalisation decreasing as countries moved towards ambulatory DOTS, although in countries such as Germany, Spain, the USA and Latvia high hospitalisation costs were still reported between 2010 and 2013 [24, 41, 52, 65] (DS-TB patients were hospitalised for an average of 115 and 72 days in Latvia and the USA, respectively). Care should therefore be taken when using costs from this review to estimate current costs for any one setting to ensure that the cost applied reflects the current mix of hospitalisation and ambulatory treatment. In particular, while the majority of MDR-TB treatment is currently provided in hospital, several countries are now piloting ambulatory models of care, so these costs may fall in coming years.

The costs of DS-TB drugs were reported in 34 studies. When compared with DS-TB treatment, drug costs remain a substantial component of MDR-TB treatment, and are particularly high in countries using individualised MDRTB regimens and/or with high levels of extensively drugresistant (XDR) TB, or not accessing concessionary prices. In the Philippines, MDR-TB drugs accounted for $46 \%$ of total provider costs in 2006 [58].

While there has been substantial research on direct costs incurred by TB patients in low- and middle-income countries, these were only included in two HIC studies, possibly highlighting a lesser interest in the poverty impact of TB in countries with higher income levels and more comprehensive social protection and health insurance systems. Nevertheless some patient costs were found. Kik et al. [29] reported that immigrant DS-TB patients in Holland paid, on average, US\$486 to receive treatment [29]. In contrast, in poorer countries, substantial attention has been paid to patient-incurred costs. Although TB treatment is provided free in many settings, it incurs a high economic burden, either through out-of-pocket/direct payments (in some settings 'under-the-counter' payments), but also through substantial productivity loss. DS-TB direct patient costs were, on average, US\$603 in UMICs, US\$84 in LMICs and US\$155 in LICs. The high values in LICs were noted by many studies to be catastrophic, and are primarily driven by costs captured in the 'other' category, which included out-of-pocket payments made by patients and their social networks for non-TB drugs, food and specialised diets, traditional healers, and accommodation, among other costs. The respective direct patient costs for MDR-TB patients were US\$660, US\$1616 and US\$406. There was substantially less evidence on the patient cost of MDR-TB. The few studies found highlight the potential of MDR-TB to have a substantially higher catastrophic impact than DS-
TB. More research is required in this area, particularly to better understand how these costs are incurred over time, and how patient cost is affected by different levels of hospitalisation.

Well defined estimates of productivity losses were included in $81 \%$ of DS-TB papers and $75 \%$ of MDR-TB papers. As a proportion of DS-TB patient costs, productivity losses comprised $96 \%$ in HICs, $68 \%$ in UMICs, $98 \%$ in LMICs and $74 \%$ in LICs. The methods used to estimate productivity loss vary widely; nevertheless, it can be seen that this is an important component of the economic impact. Therefore, by excluding this cost, the majority of reported TB patient costs are substantially underestimating the impact of TB on patients. The difference in methodological approaches taken also makes it challenging to draw general conclusions about the key drivers of patient-incurred costs, and for analysts to use this review to extrapolate patient costs across settings or over time. It is therefore recommended that, although the costs presented in this review provide some guidance, the measurement of setting-specific costs that are comprehensive may still be required in economic analyses of TB control interventions for some time to come.

We captured 90 papers in all income groups. The review of economic evaluations in TB control published by Verdier et al. [4] in 2011 included 118 papers from HICs only. This large volume was due to the inclusion of mathematical modelling papers and multiple papers using the same primary data. For MDR-TB, 16 countries were included in our review, compared with only four countries captured by Fitzpatrick and Floyd in 2012 [7]. Quality assessment is crucial for systematic reviews, but only three of the previous reviews completed this [2, 4, 5]. Using the CHEERS and TBCTA guidelines, we identified several key methodological issues that suggest further standardisation is required in order to further develop a set of costs that can be used globally. First, even when the ingredient approach to costing was used, cost items were insufficiently separated in several studies, hindering our ability to observe cost drivers and analyse trends such as drug costs over time. The lack of reporting of disaggregated costs was also an issue for patient-incurred costs. Second, methods for calculating productivity losses were not clearly explained in more than one-third of the studies that included these costs, and disparate approaches were used between studies, which led to widely different estimates within the same country. The lack of standard methods for identification, measurement and valuation of productivity losses have frequently been acknowledged in the wider literature on the measurement of costs, and minimum standardised approaches are urgently required to enable comparisons across settings, particularly in light of the increased global attention on social protection [13]. Third, even though cost 
data were collected from a relatively large number of patients, insufficient statistical analyses were undertaken in most studies. In addition to mean values, descriptive statistics, such as SD, minimum and maximum values must be presented and any outliers in the patient sample should be highlighted.

\section{Conclusion}

In summary, literature on the costs of DS-TB treatment to both providers and patients is extensive. However, evidence is still scarce on the costs of treating MDR-TB, and how these costs may vary by mode of delivery and setting. MDR-TB treatment is rapidly evolving; a recent global guideline change recommends Xpert ${ }^{\circledR}$ MTB/RIF diagnostics, which is more sensitive and also detects rifampicin resistance, therefore identifying more cases. In addition, recent global investment in further testing of existing MDRTB drugs as well as development of new drugs has been substantial. More data are urgently required to estimate the budgetary impact of these changes and to support economic evaluations of new MDR-TB control approaches.

Acknowledgments UKG and AV planned the study. YVL conducted the search, extracted, analysed and interpreted the data, and produced a draft of the manuscript. UKG also extracted, analysed and interpreted the data, and wrote components of the manuscript. AV oversaw the progression of the review, provided guidance and contributed to various versions of the manuscript. All authors read and approved the final manuscript. YVL is the overall guarantor of this work.

None of the authors have expressed any conflict of interest. Funding was received from AERAS for the conduct of this systematic literature review (Grant PHGHVK5610).

Open Access This article is distributed under the terms of the Creative Commons Attribution-NonCommercial 4.0 International License (http://creativecommons.org/licenses/by-nc/4.0/), which permits any noncommercial use, distribution, and reproduction in any medium, provided you give appropriate credit to the original author(s) and the source, provide a link to the Creative Commons license, and indicate if changes were made.

\section{References}

1. WHO. The End TB Strategy: Global strategy and targets for tuberculosis prevention, care and control after 2015. In: World Health Organisation, editor. WHO Global Tuberculosis Report. Geneva: WHO; 2014. p. 23.

2. Fryatt RJ. Review of published cost-effectiveness studies on tuberculosis treatment programmes. Int $\mathrm{J}$ Tuberc Lung Dis. 1997;1(2):101-9.

3. Russell S. The economic burden of illness for households in developing countries: a review of studies focusing on malaria, tuberculosis, and human immunodeficiency virus/acquired immunodeficiency syndrome. Am J Trop Med Hyg. 2004;71(2 Suppl):147-55.

4. Verdier JE, et al. A systematic review of economic evaluation studies of tuberculosis control in high-income countries. Int $\mathbf{J}$ Tuberc Lung Dis. 2011;15(12):1587-98.

5. Ukwaja KN, et al. The economic burden of tuberculosis care for patients and households in Africa: a systematic review. Int $\mathrm{J}$ Tuberc Lung Dis. 2012;16(6):733-9.

6. Barter DM, et al. Tuberculosis and poverty: the contribution of patient costs in sub-Saharan Africa-a systematic review. BMC Public Health. 2012;12:980.

7. Fitzpatrick C, Floyd K. A systematic review of the cost and cost effectiveness of treatment for multidrug-resistant tuberculosis. Pharmacoeconomics. 2012;30(1):63-80.

8. Diel R, et al. Costs of tuberculosis disease in the European Union: a systematic analysis and cost calculation. Eur Respir J. 2014;43(2):554-65.

9. Tanimura T, et al. Financial burden for tuberculosis patients in low- and middle-income countries: a systematic review. Eur Respir J. 2014;43(6):1763-75.

10. Google. Google translate. 201517 February 2015; electronic translation software. Available from: https://translate.google.co. uk/. Accessed 7 Feb 2015.

11. CRD. Systematic Reviews: CRD's guidance for undertaking systematic reviews in health care. In: Centre for reviews and dissemination, editor. CRD. York: University of York; 2009.

12. Shemilt I, et al. Chapter 15: incorporating economics evidence. In: Higgins J, Green S, editors. Cochrane handbook for systematic reviews of interventions. Chichester: The Cochrane Collaboration; 2011.

13. Krol M, Brouwer W, Rutten F. Productivity costs in economic evaluations: past, present, future. Pharmacoeconomics. 2013;31(7):537-49.

14. Steffen R, et al. Patients' costs and cost-effectiveness of tuberculosis treatment in DOTS and non-DOTS facilities in Rio de Janeiro, Brazil. PLoS One. 2010;5(11):e14014.

15. OANDA. Historical exchange rates. 2015: http://www.oanda. com/currency/historical-rates/. Accessed 9 Mar 2015.

16. International monetary fund, data and statistics. 2015: http:// www.imf.org/external/data.htm. Accessed 9 Mar 2015.

17. World Bank. How we classify countries. 2015; Available from: http://data.worldbank.org/about/country-and-lending-groups. Accessed 9 Mar 2015.

18. Husereau D, et al. Consolidated Health Economic Evaluation Reporting Standards (CHEERS) —explanation and elaboration: a report of the ISPOR Health Economic Evaluation Publication Guidelines Good Reporting Practices Task Force. Value Health. 2013;16(2):231-50.

19. Mauch V. The tool to estimate patients' costs, in http://www. stoptb.org/wg/dots_expansion/tbandpoverty/assets/documents/ Tool\%20to\%20estimate\%20Patients'\%20Costs.pdf. 2008, KNVI Tuberculosis Foundation. Accessed 31 Oct 2014.

20. Boyd KA, et al. Power and sample size for cost-effectiveness analysis: fFN neonatal screening. Contemp Clin Trials. 2011;32(6):893-901.

21. Hollingworth W, et al. Cost-utility analysis conducted alongside randomized controlled trials: are economic end points considered in sample size calculations and does it matter? Clin Trials. 2013;10(1):43-53.

22. Mihaylova B, et al. Review of statistical methods for analysing healthcare resources and costs. Health Econ. 2011;20(8): 897-916.

23. White VL, Moore-Gillon J. Resource implications of patients with multidrug resistant tuberculosis. Thorax. 2000;55(11):962-3.

24. Diel R, et al. Tuberculosis: cost of illness in Germany. Eur Respir J. 2012;40(1):143-51. 
25. Pooran A, et al. What is the cost of diagnosis and management of drug resistant tuberculosis in South Africa? PLoS One. 2013;8(1):e54587.

26. Dick J, Henchie S. A cost analysis of the tuberculosis control programme in Elsies River, Cape Town. S Afr Med J. 1998;88(3 Suppl):380-3.

27. Drummond M, Schulper JS, Torrance GW, O'Brien BJ, Stoddart GL. Methods for the economic evaluation of health care programmes. 3rd ed. Oxford: Oxford University Press; 2005.

28. Rajbhandary SS, Marks SM, Bock NN. Costs of patients hospitalized for multidrug-resistant tuberculosis. Int J Tuberc Lung Dis. 2004;8(8):1012-6.

29. Kik SV, et al. Direct and indirect costs of tuberculosis among immigrant patients in the Netherlands. BMC public health. 2009;9:283.

30. Jacobs B, et al. Cost-effectiveness analysis of the russian treatment scheme for tuberculosis versus short-course chemotherapy: results from Tomsk, Siberia. Int J Tuberc Lung Dis. 2002;6(5):396-405.

31. Kang YA, et al. Cost of treatment for multidrug-resistant tuberculosis in South Korea. Respirology. 2006;11(6):793-8.

32. Pantoja A, et al. Economic evaluation of public-private mix for tuberculosis care and control, India. Part I. Socio-economic profile and costs among tuberculosis patients. Int J Tuberc Lung Dis. 2009;13(6):698-704.

33. Weis SE, et al. Treatment costs of directly observed therapy and traditional therapy for Mycobacterium tuberculosis: a comparative analysis. Int J Tuberc Lung Dis. 1999;3(11):976-84.

34. Floyd K, et al. Cost and cost-effectiveness of PPM-DOTS for tuberculosis control: evidence from India. Bull World Health Organ. 2006;84(6):437-45.

35. Vassall A, et al. Reforming tuberculosis control in Ukraine: results of pilot projects and implications for the national scaleup of DOTS. Health Policy Plan. 2009;24(1):55-62.

36. Floyd K, et al. Cost and cost-effectiveness of increased community and primary care facility involvement in tuberculosis care in Lilongwe District, Malawi. Int J Tuberc Lung Dis. 2003;7(9 Suppl 1):S29-37.

37. Okello D, et al. Cost and cost-effectiveness of community-based care for tuberculosis patients in rural Uganda. Int J Tuberc Lung Dis. 2003;7(9 Suppl 1):S72-9.

38. Burman WJ, et al. A cost-effectiveness analysis of directly observed therapy vs self-administered therapy for treatment of tuberculosis. Chest. 1997;112(1):63-70.

39. Burns AD, Harrison AC. Costs of investigating and managing non-residents with possible tuberculosis: New Zealand experience of an international problem. Respirology. 2007;12(2):262-6.

40. Atun RA, et al. Costs and outcomes of tuberculosis control in the Russian Federation: retrospective cohort analysis. Health Policy Plan. 2006;21(5):353-64.

41. Miller TL, et al. The value of effective public tuberculosis treatment: an analysis of opportunity costs associated with multidrug resistant tuberculosis in Latvia. Cost Eff Resour Alloc. 2013;11(1):9.

42. Fairall L, et al. Cost-effectiveness of educational outreach to primary care nurses to increase tuberculosis case detection and improve respiratory care: economic evaluation alongside a randomised trial. Trop Med Int Health. 2010;15(3):277-86.

43. Masobe P, Lee T, Price M. Isoniazid prophylactic therapy for tuberculosis in HIV-seropositive patients-a least-cost analysis. S Afr Med J. 1995;85(2):75-81.

44. Cusmano LG, et al. Study of the intangible costs and impact of the household component in the social cost of tuberculosis. Revista Argentina de Salud Publica. 2009;1(1):18-23.

45. Moalosi G, et al. Cost-effectiveness of home-based care versus hospital care for chronically ill tuberculosis patients,
Francistown, Botswana. Int J Tuberc Lung Dis. 2003;7(9 Suppl 1):S80-5.

46. Vassall A, et al. Cost-effectiveness of different treatment strategies for tuberculosis in Egypt and Syria. Int J Tuberc Lung Dis. 2002;6(12):1083-90.

47. Khan MA, et al. Costs and cost-effectiveness of different DOT strategies for the treatment of tuberculosis in Pakistan. Directly Observed Treatment. Health Policy Plan. 2002;17(2):178-86.

48. Bocchino M, et al. Cost determinants of tuberculosis management in a low-prevalence country. Int $\mathbf{J}$ Tuberc Lung Dis. 2006;10(2):146-52.

49. Wilkinson D, Floyd K, Gilks CF. Costs and cost-effectiveness of alternative tuberculosis management strategies in South Africaimplications for policy. S Afr Med J. 1997;87(4):451-5.

50. Saunderson PR. An economic evaluation of alternative programme designs for tuberculosis control in rural Uganda. Soc Sci Med. 1995;40(9):1203-12.

51. Eralp MN, et al. Screening of healthcare workers for tuberculosis: development and validation of a new health economic model to inform practice. BMJ Open. 2012;2(2):e000630.

52. Miller TL, et al. The societal cost of tuberculosis: Tarrant County, Texas, 2002. Ann Epidemiol. 2010;20(1):1-7.

53. Peralta Perez M, et al. Costo del programma nacional de control de la tuberculosis. Centro provincial de higiene y epidemiologia de ciudad de La Habana, 2002. Rev Cuba Med Trop. 2006;58(1):63-7.

54. El-Sony AI. The cost to health services of human immunodeficiency virus (HIV) co-infection among tuberculosis patients in Sudan. Health Policy. 2006;75(3):272-9.

55. Floyd K, et al. Cost and cost-effectiveness of multidrug-resistant tuberculosis treatment in Estonia and Russia. Eur Respir J. 2012;40(1):133-42.

56. Marks SM, et al. Treatment practices, outcomes, and costs of multidrug-resistant and extensively drug-resistant tuberculosis, United States, 2005-2007. Emerg Infect Dis. 2014;20(5): 812-21.

57. Schnippel K, et al. Costs of inpatient treatment for multi-drugresistant tuberculosis in South Africa. Trop Med Int Health. 2013;18(1):109-16.

58. Tupasi TE, et al. Feasibility and cost-effectiveness of treating multidrug-resistant tuberculosis: a cohort study in the Philippines. PLoS Med. 2006;3(9):e352.

59. Pichenda K, et al. Non-hospital DOT and early diagnosis of tuberculosis reduce costs while achieving treatment success. Int J Tuberc Lung Dis. 2012;16(6):828-34.

60. Xu Q, Jin SG, Zhang LX. Cost effectiveness of DOTS and nonDOTS strategies for smear-positive pulmonary tuberculosis in Beijing. Biomed Environ Sci. 2000;13(4):307-13.

61. Kamolratanakul P, et al. Cost analysis of different types of tuberculosis patient at tuberculosis centers in Thailand. Southeast Asian J Trop Med Public Health. 2002;33(2):321-30.

62. Suarez PG, et al. Feasibility and cost-effectiveness of standardised second-line drug treatment for chronic tuberculosis patients: a national cohort study in Peru. Lancet. 2002;359(9322):1980-9.

63. Pan HQ, et al. Analysis of the economic burden of diagnosis and treatment of tuberculosis patients in rural China. Int $\mathrm{J}$ Tuberc Lung Dis. 2013;17(12):1575-1580+i.

64. Jacquet V, et al. Impact of DOTS expansion on tuberculosis related outcomes and costs in Haiti. BMC Public Health. 2006;6:209.

65. Montes-Santiago J, et al. Tuberculosis-related hospitalization in Spain: a cost analysis. Enferm Infec Microbiol Clin. 2010;28(6):358-61.

66. Nganda B, et al. Cost and cost-effectiveness of increased community and primary care facility involvement in tuberculosis 
care in Machakos District, Kenya. Int $\mathrm{J}$ Tuberc Lung Dis. 2003;7(9 Suppl 1):S14-20.

67. Wyss K, Kilima P, Lorenz N. Costs of tuberculosis for households and health care providers in Dar es Salaam, Tanzania. Trop Med Int Health. 2001;6(1):60-8.

68. Mauch V, et al. Free tuberculosis diagnosis and treatment are not enough: patient cost evidence from three continents. Int $\mathrm{J}$ Tuberc Lung Dis. 2013;17(3):381-7.

69. Aye R, et al. Household costs of illness during different phases of tuberculosis treatment in Central Asia: a patient survey in Tajikistan. BMC Public Health. 2010;10:18.

70. Jackson S, et al. Poverty and the economic effects of TB in rural China. Int J Tuberc Lung Dis. 2006;10(10):1104-10.

71. Yitayal M, et al. Assessment of cost of tuberculosis to patients and their families: a cross-sectional study at Addet Health Center, Yilmana Densa District, Amhara National Regional State. Ethiop Med J. 2014;Suppl 1:23-30.

72. Elamin EI, et al. Cost of illness of tuberculosis in Penang, Malaysia. Pharm World Sci. 2008;30(3):281-6.

73. Islam MA, et al. Cost-effectiveness of community health workers in tuberculosis control in Bangladesh. Bull World Health Organ. 2002;80(6):445-50.

74. Vassall A, et al. Patient costs of accessing collaborative tuberculosis and human immunodeficiency virus interventions in Ethiopia. Int J Tuberc Lung Dis. 2010;14(5):604-10.

75. Gospodarevskaya E, et al. Patient costs during tuberculosis treatment in Bangladesh and Tanzania: The potential of shorter regimens. Int J Tuberc Lung Dis. 2014;18(7):810-817+i-ii.

76. Migliori GB, et al. Cost-effectiveness analysis of tuberculosis control policies in Ivanovo Oblast, Russian Federation. Ivanovo Tuberculosis Project Study Group. Bull World Health Organ. 1998;76(5):475-83

77. Sawert H, et al. Costs and benefits of improving tuberculosis control: the case of Thailand. Soc Sci Med. 1997;44(12):1805-16.

78. Migliori GB, et al. Cost-comparison of different management policies for tuberculosis patients in Italy. AIPO TB Study Group. Bull World Health Organ. 1999;77(6):467-76.

79. Mahendradhata $Y$, et al. The incremental cost-effectiveness of engaging private practitioners to refer tuberculosis suspects to DOTS services in Jogjakarta, Indonesia. Am J Trop Med Hyg. 2010;82(6):1131-9.

80. Prado TN, et al. Cost-effectiveness of community health worker versus home-based guardians for directly observed treatment of tuberculosis in Vitoria, Espirito Santo State, Brazil. Cad Saude Publica. 2011;27(5):944-52.

81. Costa JG, et al. Tuberculosis in Salvador, Brazil: costs to health system and families. Rev Saude Publica. 2005;39(1):122-8.

82. John KR, et al. Costs incurred by patients with pulmonary tuberculosis in rural India. Int $J$ Tuberc Lung Dis. 2009;13(10):1281-7.

83. Muniyandi $\mathrm{M}$, et al. A comparison of costs to patients with tuberculosis treated in a DOTS programme with those in a nonDOTS programme in South India. $J$ Health Manag. 2008;10(1):9-24.

84. Datiko DG, Lindtjorn B. Cost and cost-effectiveness of smearpositive tuberculosis treatment by Health Extension Workers in Southern Ethiopia: a community randomized trial. PLoS One. 2010;5(2):e9158.

85. Wandwalo E, Robberstad B, Morkve O. Cost and cost-effectiveness of community based and health facility based directly observed treatment of tuberculosis in Dar es Salaam, Tanzania. Cost Eff Resour Alloc. 2005;3:6.

86. Rouzier VA, et al. Patient and family costs associated with tuberculosis, including multidrug-resistant tuberculosis, Ecuador. Int J Tuberc Lung Dis. 2010;14(10):1316-22.
87. Diel R, et al. Cost of multi drug resistance tuberculosis in Germany. Respir Med. 2014;108(11):1677-87.

88. Wurtz R, White WD. The cost of tuberculosis: utilization and estimated charges for the diagnosis and treatment of tuberculosis in a public health system. Int $\mathbf{J}$ Tuberc Lung Dis. 1999;3(5):382-7.

89. Maponga CC, Nazerali H, Mungwindri C, Wingwirl M. Cost implications of the tuberculosis/HIV co-epidemic and drug treatment of tuberculosis in Zimbabwe. J Soc Adm Pharm. 1996;13(1):20-9.

90. DeRiemer K, et al. Does DOTS work in populations with drugresistant tuberculosis? Lancet. 2005;365(9466):1239-45.

91. Dye C, Espinal MA. Will tuberculosis become resistant to all antibiotics? Proc Biol Sci. 2001;268(1462):45-52.

92. Palmer CS, et al. A model of the cost-effectiveness of directly observed therapy for treatment of tuberculosis. J Public Health Manag Pract. 1998;4(3):1-13.

93. Marchand R, Tousignant $\mathrm{P}$, Chang $\mathrm{H}$. Cost-effectiveness of screening compared to case-finding approaches to tuberculosis in long-term care facilities for the elderly. Int $\mathrm{J}$ Epidemiol. 1999;28(3):563-70.

94. MacIntyre CR, Plant AJ, Hendrie D. Shifting the balance between in-patient and out-patient care for tuberculosis results in economic savings. Int J Tuberc Lung Dis. 2001;5(3):266-71.

95. Tu HZ, et al. Combination of molecular assay and clinical evaluation for early confirmation of tuberculosis cases. Clin Microbiol Infect. 2011;17(5):712-4.

96. Ruiz MG, Nunez LR, Lezama MAS, Valle FC. Costos de atencipn de la tuberculosis: caso del instituto nacional de enfermedadaes respiratorias (INER). Rev Instituto Nac Enferm Respir. 2003:16(4):219-25.

97. Sinanovic E, Kumaranayake L. Financing and cost-effectiveness analysis of public-private partnerships: provision of tuberculosis treatment in South Africa. Cost Eff Resour Alloc. 2006;4:11.

98. Jackson S, et al. Poverty and the economic effects of TB in rural China. Int J Tuberc Lung Dis. 2006;10(10):1104-10.

99. Liu X, et al. How affordable are tuberculosis diagnosis and treatment in rural China? An analysis from community and tuberculosis patient perspectives. Trop Med Int Health. 2007;12(12):1464-71.

100. Guzman-Montes GY, Ovalles RH, Leniado-Laborin R. Indirect patient expenses for antituberulosis treatment in Tijuana, Mexico: is treatment really free? J Infect Dev Ctries. 2009;3(10):778-82.

101. Samandari T, et al. Costs and consequences of additional chest $\mathrm{X}$-ray in a tuberculosis prevention program in Botswana. Am J Respir Crit Care Med. 2011;183(8):1103-11.

102. Nieto E, et al. Cost-effectiveness of an alternative tuberculosis treatment: home-based guardian monitoring of patients. Rev Panam Salud Publica. 2012;32(3):178-84.

103. Zou G, et al. Incremental cost-effectiveness of improving treatment results among migrant tuberculosis patients in Shanghai. Int J Tuberc Lung Dis. 2013;17(8):1056-64.

104. Wei X, et al. China tuberculosis policy at crucial crossroads: Comparing the practice of different hospital and tuberculosis control collaboration models using survey data. PLoS One. 2014:9(3):e90596.

105. Foster N, et al. The economic burden of TB diagnosis and treatment in South Africa. Soci Sci Med. 2015;130:42-50.

106. Rajeswari R, et al. Socio-economic impact of tuberculosis on patients and family in India. Int $\mathbf{J}$ Tuberc Lung Dis. 1999;3(10):869-77.

107. Peabody JW, et al. The burden of disease, economic costs and clinical consequences of tuberculosis in the Philippines. Health Policy Plan. 2005;20(6):347-53. 
108. Aspler A, et al. Cost of tuberculosis diagnosis and treatment from the patient perspective in Lusaka, Zambia. Int $\mathbf{J}$ Tuberc Lung Dis. 2008;12(8):928-35.

109. Mauch V, et al. Assessing access barriers to tuberculosis care with the tool to estimate patients' costs: pilot results from two districts in Kenya. BMC Public Health. 2011;11:43.

110. Umar NA, et al. Direct costs of pulmonary tuberculosis among patients receiving treatment in Bauchi State, Nigeria. Int J Tuberc Lung Dis. 2012;16(6):835-40.

111. Gibson N, Boillot F, Jalloh H. The cost of tuberculosis to patients in Sierra Leone's war zone. Int $\mathrm{J}$ Tuberc Lung Dis. 1998;2(9):726-31.
112. Karki DK, et al. Costs of a successful public-private partnership for TB control in an urban setting in Nepal. BMC public health. 2007;7:84.

113. Mirzoev TN, et al. Community-based DOTS and family member DOTS for TB control in Nepal: costs and cost-effectiveness. Cost Eff Resour Alloc. 2008;6:20.

114. Laokri S, et al. Patients are paying too much for tuberculosis: a direct cost-burden evaluation in Burkina Faso. PLoS One. 2013;8(2):e56752.

115. Laokri S, et al. A care pathway analysis of tuberculosis patients in Benin: highlights on direct costs and critical stages for an evidence-based decision-making. PLoS One. 2014:9(5):e96912. 